\title{
Short- and midterm outcomes of coronary artery bypass surgery performed by surgeons in training
}

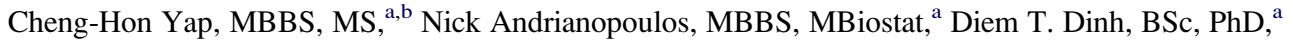 \\ Baki Billah, BSc, MSc, MAS, PhD, ${ }^{a}$ Alexander Rosalion, FRACS, ${ }^{\mathrm{c}}$ Julian A. Smith, FRACS, ${ }^{\text {d,f }}$ Gilbert C. Shardey, FRACS, \\ Peter D. Skillington, FRACS, ${ }^{\mathrm{b}}$ James Tatoulis, FRACS, MD, ${ }^{\mathrm{b}}$ Morteza Mohajeri, FRACS, ${ }^{\mathrm{e}}$ Michael Yii, FRACS, MS, \\ and Chistopher M. Reid, $\mathrm{PhD}^{\mathrm{a}}$
}

Objective: The effect of training on outcomes in cardiac surgery is poorly studied. We aimed to study the results of coronary artery bypass grafting procedures performed by surgeons in training across our state with respect to short- and midterm postoperative outcomes.

\begin{abstract}
Methods: All coronary artery bypass grafting surgeries performed by trainee surgeons between July 2001 and December 2006 were compared with those performed by consultant surgeons using mandatory prospectively collected statewide data. Early mortality; prolonged ventilation or intensive care unit stay; return to operating theater for bleeding, stroke, myocardial infarction, or renal failure; and 5-year survival were compared using propensity score analysis.

Results: A total of 7745 surgeries were included in this study. Trainees performed 983 (13\%) surgeries. Trainee surgeries had longer perfusion and crossclamp times. Crude early postoperative outcomes were similar between trainee and consultant surgeries. After propensity score adjustment, early outcomes remained similar, with the exception of myocardial infarction $(0.8 \%$ in trainee surgeries vs $0.4 \%$ in consultant surgeries, $P=.046)$. Adjusted 1-, 3-, and 5-year survivals were similar between trainee and consultant surgeries: 95.3\% versus $95.5 \%, 90.8 \%$ versus $92.0 \%$, and $86.3 \%$ versus $87.1 \%$, respectively.
\end{abstract}

Conclusion: Coronary artery bypass grafting performed by trainee surgeons within a supervised program is safe with acceptable short- and midterm outcomes.

Earn CME credits at

http://cme.ctsnetjournals.org

Training residents in cardiac surgery poses many potential concerns. Given the complexities and lack of minor cases in cardiac surgery, training cardiac surgeons has the potential to lead to longer operating times and poorer patient outcomes. However, the need to train future cardiac surgeons requires trainees to operate under appropriate levels of supervision. Hence, a tension exists within cardiac surgical units and between individual consultant surgeons to strike a balance between attaining the highest possible standard of care for patients and the duty to train future surgeons. ${ }^{1}$

The volume of surgeries performed by individual surgeons ${ }^{2}$ has been shown to be an important determinant of

From the Department of Epidemiology and Preventive Medicine, Monash University, Victoria, Australia; Department of Cardiothoracic Surgery, The Royal Melbourne Hospital, ${ }^{\text {b }}$ Victoria, Australia; Department of Cardiothoracic Surgery, St Vincent's Hospital Melbourne, ${ }^{c}$ Victoria, Australia; Department of Cardiothoracic Surgery, Monash Medical Centre, ${ }^{\mathrm{d}}$ Victoria, Australia; The Geelong Hospital, ${ }^{\mathrm{e}}$ Victoria, Australia; and Department of Surgery, ${ }^{\mathrm{f}}$ Monash University, Victoria, Australia.

Received for publication July 1, 2008; revisions received Sept 8, 2008; accepted for publication Oct 9, 2008.

Address for reprints: Cheng-Hon Yap, MBBS, MS, Department of Cardiothoracic Surgery, The Royal Melbourne Hospital, Parkville, Victoria 3050 Australia (E-mail: chenghonyap@hotmail.com).

J Thorac Cardiovasc Surg 2009; 137:1088-92

$0022-5223 / \$ 36.00$

Copyright (c) 2009 by The American Association for Thoracic Surgery doi:10.1016/j.jtcvs.2008.10.011 patient outcomes. The rise in database participation for the monitoring of outcomes in cardiac surgery and the increasing interest in public reporting of cardiac surgical outcomes have resulted in high levels of scrutiny of individual cardiac surgeon's outcomes. ${ }^{3,4}$ There is concern that these factors may lead to less opportunity and experience for trainee surgeons. The recent moves to reduce working hours of junior medical staff without a commensurate increase in the intensity and efficiency of training programs has the potential to compound this issue. ${ }^{5}$

In light of these concerns, it is important to study the effect of current surgical training on outcomes in cardiac surgery. There has been little attention given to this subject to date with studies being limited to single institutions and to an analysis of only early postoperative outcomes. ${ }^{6-13}$ We aimed to study the impact of trainee cardiac surgeons performing coronary artery bypass grafting (CABG) procedures across 6 institutions with respect to early postoperative outcomes and midterm survival.

\section{METHODS \\ Data Collection}

First-time isolated CABG surgeries performed by trainee or consultant surgeons between July 1, 2001 and December 31, 2006 were included in the study. Patients undergoing emergency procedures or those who have had previous cardiac surgery were excluded. Data were prospectively collected as part of the Australasian Society of Cardiac and Thoracic Surgeons (ASCTS) database project. This is a statewide data collection project of cardiac surgery procedures in the state of Victoria, Australia with mandatory participation of all 6 government-funded adult cardiac surgical institutions. ${ }^{14}$ The participating institutions during the study period were Royal 

Abbreviations and Acronyms
ASCTS $=$ Australasian Society of Cardiac and Thoracic Surgeons
$\mathrm{CABG}=$ coronary artery bypass grafting
$\mathrm{CI}=$ confidence interval
ICU = intensive care unit
MI = myocardial infarction
$\mathrm{OR} \quad=$ odds ratio

Melbourne Hospital, the Alfred Hospital, Monash Medical Centre, Geelong Hospital, Austin and Repatriation Medical Centre, and St Vincent's Hospital Melbourne. Four of the 6 hospitals involved in the study had a single cardiac surgery trainee post during the entire study period. One hospital had 2 trainee posts during the entire study period, and 1 hospital was not a training hospital. Individual trainee appointments to hospitals are usually for only 1 year and never exceeded 2 years.

The ASCTS database contained detailed information on patient demographics, preoperative risk factors, operative details, postoperative hospital course, and morbidity and mortality outcomes. This data were collected prospectively using an agreed data set and definitions ${ }^{15}$ as part of clinical care by surgeons, perfusionist, resident medical officers, and database managers. Thirty-day mortality information was obtained by telephone contact with patient, family member, or medical practitioner. The data were subject to external audit measures with an overall data accuracy of $97.4 \%$ recently reported. ${ }^{16}$ The institutional review board of each participating hospital had previously approved the use of these databases for research and hence, the need for individual patient consent was waived for this study.

Midterm survival status of patients was obtained from the Australian National Death Index. The closing date was December 31, 2006.

\section{Study End Points}

The outcomes of surgeries performed by trainee surgeons were compared to those performed by consultant surgeons. Trainee surgeries were those in which the trainee performed the entire operation as the primary surgeon or the majority of the critical parts of the operations, such as most of the distal coronary anastomoses. In the former situation, consultant supervision varied depending on the level of training of the trainee surgeon, ranging from the consultant surgeon assisting the trainee throughout the surgeries to the consultant surgeon being available, but not directly present in the operating room. Our primary study end points, defined before analysis, were 7 early postoperative serious events. These were 30-day mortality; prolonged ventilation (greater than 24 hours); intensive care unit (ICU) stay greater than 21 hours ( 21 hours is the median ICU stay duration); return to the operating theater for bleeding, myocardial infarction (MI), stroke; and new renal failure. MI was defined as at least 2 of the following: cardiac enzyme elevation (creatinine kinase-myocardial band [CK-MB] $>30 \mathrm{U} / \mathrm{L}$ or troponin $>20$ $\mu \mathrm{g} / \mathrm{L})$, new wall motion abnormalities, and new $\mathrm{Q}$ waves on at least 2 serial electrocardiograms. New renal failure was defined as at least 2 of the following: serum creatinine increased to $>0.2 \mathrm{mmol} / \mathrm{L}$, doubling or greater increase in serum creatinine over the preoperative value, and a new requirement for renal replacement therapy. Our secondary end point was midterm survival.

\section{Statistical Analysis}

Descriptive data are expressed as the mean \pm standard deviation. The level of statistical significance $(\alpha)$ was set at .05 (two-sided). Baseline characteristics, operative factors, and univariate outcomes were compared between surgeries performed by trainee surgeons and those by consultant surgeons with 2 -sample tests. The Wilcoxon rank-sum test and the chi-square test were used for group comparisons among continuous and categorical variables, respec- tively. To control for confounding, a propensity score was calculated from a logistic regression as the probability of the surgery being performed by a trainee surgeon, given all the baseline preoperative variables. Variables entered into the propensity model were age; sex; obesity; current smoker; history of diabetes; hypercholesterolemia; hypertension; cerebrovascular disease; peripheral vascular disease; renal failure; respiratory disease; recent MI; congestive heart failure; unstable angina; New York Heart Association class; Canadian Cardiovascular Class; 3-vessel disease; severe left ventricular impairment; left main stenosis; use of intravenous nitrates, aspirin, clopidogrel, anticoagulants, or steroids at time of surgery; recent thrombolysis; and urgent status. The propensity score and the surgeon variable were then forced into a logistic regression model for each end point studied to obtain the adjusted odds ratio (OR) for the trainee surgeon status. Kaplan-Meier analysis was used to assess midterm survival. Midterm survival was further adjusted for the propensity score by incorporating the propensity score and surgeon status into a Cox-proportional hazard model. Differences in midterm survival were assessed by the log-rank tests. Stata version 9.2 (StatCorp, College Station, TX) was used for statistical analysis.

\section{RESULTS}

A total of 7745 CABG surgeries were included in this study. Trainees performed $983(12.7 \%)$ surgeries. Table 1 shows the patient characteristics. There were some

TABLE 1. Preoperative characteristics of patients undergoing surgery by trainee and consultant surgeons

\begin{tabular}{|c|c|c|c|}
\hline Variable & $\begin{array}{l}\text { Trainee } \\
(\mathbf{n}=\mathbf{9 8 3})\end{array}$ & $\begin{array}{l}\text { Consultant } \\
(n=6762)\end{array}$ & $\boldsymbol{P}$ \\
\hline Age (mean +SD) & $65.7+9.6$ & $65.8+10.4$ & .73 \\
\hline Males (\%) & 76.9 & 76.4 & .75 \\
\hline \multicolumn{4}{|l|}{ Risk factors } \\
\hline Current smoker (\%) & 22.5 & 20.1 & .14 \\
\hline Diabetes $(\%)$ & 30.3 & 33.4 & .05 \\
\hline Hypercholesterolemia (\%) & 80.5 & 82.0 & .26 \\
\hline Hypertension $(\%)$ & 76.7 & 74.2 & .09 \\
\hline Cerebrovascular disease $(\%)$ & 9.7 & 11.6 & .07 \\
\hline Peripheral vascular disease $(\%)$ & 12.8 & 13.8 & .41 \\
\hline Renal failure (\%) & 1.0 & 2.8 & .004 \\
\hline Respiratory disease $(\%)$ & 88.2 & 86.7 & .20 \\
\hline \multicolumn{4}{|l|}{ Cardiac history } \\
\hline Previous PCI (\%) & 10.7 & 10.7 & .97 \\
\hline $\begin{array}{l}\text { Myocardial infarction } \\
\text { within } 21 \mathrm{~d}(\%)\end{array}$ & 32.6 & 33.3 & .77 \\
\hline $\begin{array}{l}\text { History of congestive heart } \\
\text { failure }(\%)\end{array}$ & 17.0 & 20.0 & .02 \\
\hline Unstable angina $(\%)$ & 28.1 & 29.6 & .35 \\
\hline NYHA (\%) class III-IV & 22.7 & 26.9 & $<.001$ \\
\hline \multicolumn{4}{|l|}{ Number of diseased vessels (\%) } \\
\hline 1 & 5.3 & 4.3 & $<.001$ \\
\hline 2 & 28.1 & 20.4 & $<.001$ \\
\hline 3 & 66.6 & 75.3 & $<.001$ \\
\hline Left main stenosis $>50 \%(\%)$ & 24.6 & 23.2 & .33 \\
\hline \multicolumn{4}{|l|}{ Left ventricular function $(\%)$} \\
\hline Normal $(E F>60 \%)$ & 55.8 & 49.3 & $<.001$ \\
\hline Mild impairment (EF 46\%-60\%) & 27.4 & 29.7 & $<.001$ \\
\hline $\begin{array}{l}\text { Moderate impairment } \\
\quad(\text { EF } 30 \%-45 \%)\end{array}$ & 14.8 & 16.0 & $<.001$ \\
\hline Severe impairment $(\mathrm{EF}<30 \%)$ & 2.0 & 5.0 & $<.001$ \\
\hline
\end{tabular}

$P C I$, Percutaneous coronary intervention; NYHA, New York Heart Association; $E F$, ejection fraction; $S D$, standard deviation; $E F$, ejection fraction. 
TABLE 2. Operative variables for trainee and consultant surgeries

\begin{tabular}{lccc}
\hline \multicolumn{1}{c}{ Variable } & Trainee & Consultant & $\boldsymbol{P}$ \\
\hline Off-pump operation (\%) & 7.6 & 8.2 & .53 \\
Perfusion time (min), mean & 95.7 & 89.3 & $<.001$ \\
Perfusion time/distal anastomoses & 31.8 & 26.6 & $<.001$ \\
$\quad$ (min), mean & & & \\
Crossclamp time (min), mean & 74.7 & 65.9 & $<.001$ \\
Crossclamp time/distal anastomoses & 24.5 & 19.4 & $<.001$ \\
$\quad$ (min), mean & & & \\
Internal mammary artery used & 98.2 & 97.2 & .08 \\
No. distal anastomoses (mean) & 3.0 & 3.3 & $<.001$ \\
No. of distal anastamoses (\%) & & & \\
$\quad$ X1 & 3.9 & 3.2 & .29 \\
X2 & 24.7 & 15.1 & $<.001$ \\
X3 & 43.7 & 39.8 & .02 \\
>X3 & 27.7 & 41.9 & $<.001$ \\
\hline
\end{tabular}

important differences in the patient characteristics between the trainee and consultant groups. Trainees were less likely to operate on patients with renal failure, a history of congestive heart failure, advanced New York Heart Association class, or those with 3-vessel disease and subnormal left ventricular ejection fraction. Table 2 displays important differences in operative variables. Most surgeons in this study use a single aortic crossclamp technique (partial occlusion clamp not used for proximal anastomoses), which is reflected in these results of crossclamp and perfusion times. Trainee surgeries had longer perfusion and crossclamp times. When adjusted for the number of distal anastomoses performed, trainees on average took 5 minutes longer for each distal anastomosis. Trainees performed surgeries with fewer distal anastomoses compared with consultant surgeries $(3.0$ vs $3.3, P<.001)$. There were no significant differences with regards to the use of off-pump CABG or internal thoracic artery usage.

There were no differences in the incidence of the primary end points studied between trainee and consultant surgeries, as shown in Table 3 . Thirty-day mortality was $1.8 \%$ and $1.4 \%(P=0.32)$ and $\mathrm{MI}$ rate was $0.8 \%$ and $0.4 \%(P=$ $.06)$ for trainee and consultant surgeries, respectively. The propensity score model constructed performed well with a c-index of 0.74 . The model balanced the variables well across propensity quartiles, with 89 of 104 variables being similar between groups. After adjusting for the propensity score, trainee status was significantly associated with MI (OR 2.26, 95\% confidence interval [CI] 1.02 to $5.02, P=$ $.046)$. There were no differences in the adjusted incidence of 30-day mortality, prolonged ventilation or ICU stay, return to operating theater for bleeding, stroke, or new renal failure (Table 3).

Midterm survival data were available on the 7044 $(90.9 \%)$ of study patients. Of these, trainees had performed $912(13.0 \%)$ and consultants had performed $6132(87.0 \%)$ of the surgeries. At a median postoperative interval of 1287 days or 3.5 years, overall crude survival was similar at $93.4 \%$ of trainee surgeries and $93.6 \%$ of consultant surgeries $(P=.94)$. When adjusted for propensity score, the trainee status was not associated with midterm survival (hazard ratio $1.03,95 \%$ CI 0.79 to $1.35, P=.83$ ). Kaplan-Meier survival curves, adjusted for the propensity score, for the 2 groups are shown in Figure 1. There were no significant differences in adjusted survival at 1,3 , and 5 years between trainee and consultant surgeries: $95.3 \%$ versus $95.5 \%, 90.8 \%$ versus $92.0 \%$, and $86.3 \%$ versus $87.1 \%$, respectively.

\section{DISCUSSION}

We have shown that the current system of trainee surgeons performing $\mathrm{CABG}$ procedures in a closely supervised environment in accordance with trainees' level of training allows for patient outcomes that are acceptable in comparison to surgeries performed by consultant surgeons. The Australian system of training, overseen by the Royal Australasian College of Surgeons, allows for 4 core years of cardiothoracic training, during which a trainee is expected to assume increasing levels of responsibility for leading cardiac operations. In general, trainees in cardiac surgery have undergone a minimum of 3 resident medical officer years in surgery following an intern year, 2 years as general surgery trainees, and then 4 trainee years in cardiac surgery. Cardiac surgery trainees learn the component procedures of conduit harvest, sternotomy, and cannulation in their first 2 years and perform entire operations in their third and fourth years. By the fourth year of training, the trainee is expected to be able to perform CABG procedures without the consultant surgeon directly assisting the trainee. This system is similar

TABLE 3. Study outcomes for trainee and consultant surgeries

\begin{tabular}{lccccc}
\hline \multicolumn{1}{c}{ Outcome } & Trainee & Consultant & $\boldsymbol{P}$ & Adjusted OR (95\% CI) \\
\hline Mortality & $18(1.8)$ & $96(1.4)$ & .32 & $1.48(0.89-2.46)$ \\
Ventilation $>24 \mathrm{~h}$ & $82(8.3)$ & $492(7.3)$ & .23 & $1.21(0.94-1.54)$ & .13 \\
ICU $>$ 21 h & $500(51.3)$ & $3622(53.9)$ & .14 & $0.93(0.82-1.07)$ & .13 \\
RTT for bleeding & $23(2.3)$ & $160(2.4)$ & .96 & $.02(0.65-1.59)$ & .32 \\
MI & $8(0.8)$ & $26(0.4)$ & .06 & $.26(1.02-5.02)$ & $.53(0.87-2.68)$ \\
Stroke & $15(1.5)$ & $74(1.1)$ & .23 & .96 & .046 \\
New renal failure & $37(3.8)$ & $252(3.7)$ & .96 & $.14-1.55)$ & .63 \\
\hline
\end{tabular}

Percentages are given in parentheses. $O R$, Odds ratio; $C I$, confidence interval; $I C U$, intensive care unit; $R T T$, return to operating theater; $M I$, myocardial infarction. 


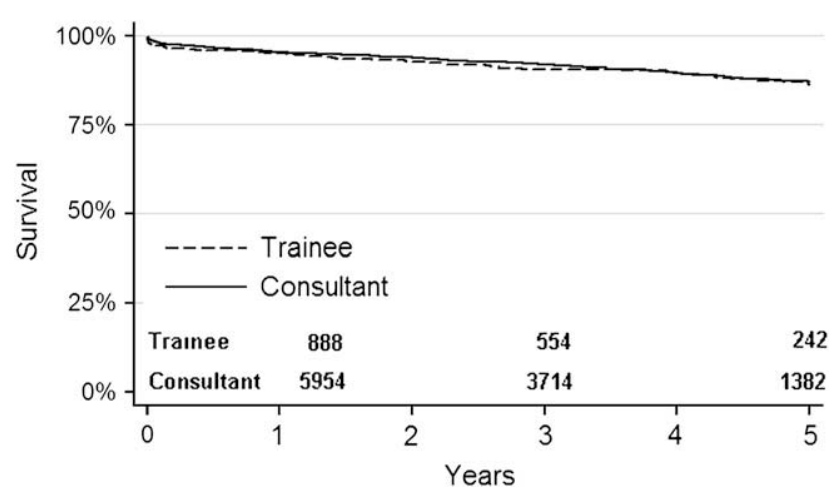

FIGURE 1. Five-year survival curve, adjusted for propensity score, for patients undergoing coronary artery bypass grafting by trainee and consultant surgeons.

to most European and North American training systems. It relies on the judgment of the supervising surgeon to resolve the tension between achieving the highest standard of care for each individual patient and the requirement to train future surgeons. The trainee surgeries are thus likely to be, on average, lower-risk surgeries, which is reflected in the lower incidence of several comorbidities related to the severity of coronary artery disease such as diabetes and renal failure, the lower incidence of left ventricular impairment, and the fewer number of coronary grafts.

The MI rate in trainee surgeries was $0.8 \%$ compared with $0.4 \%$ in consultant surgeries. This difference was not significant in an unadjusted comparison. However, with adjustment for differences in patient characteristics using propensity scoring, this difference was significant. Nevertheless, the MI rate in the trainee group of $<1.0 \%$ is comparable to if not better than other centers. ${ }^{6,8,12,17,18}$ Importantly, it was not associated with an increase in either early or midterm mortality.

There is increased scrutiny of cardiac surgical results by the public and payers, with publication of individual surgeon and institutional results in several health care systems. ${ }^{3,4}$ In this climate, it is incumbent on the surgical profession to uphold the culture of training ${ }^{1}$ within our tertiary hospital institutions while maintaining a high level of patient care. Studies such as this and others ${ }^{6,8,11,13}$ are important to audit trainee performance and to reassure the public and payers that good patient outcomes are being achieved. Importantly, we are able to show that in addition to acceptable early postoperative outcomes, midterm survivals of trainee-performed surgeries are good at $86 \%$, and not different to consultant surgeries. Such evaluation of midterm outcomes is vital as small variations in the quality of surgical care may not be manifest in the early postoperative period.

Our findings are consistent with studies from various European and North American institutions. ${ }^{6-13}$ These studies are mostly single-institution analyses that show no significant increase in early morbidity and mortality among trainee surger- ies. However, such single-institution studies may not be representative of the real world, as they may be derived from centers of excellence and subject to strong publication bias. The only 2 multi-institutional studies are from the Department of Veterans Affairs Cooperative Study on Valvular Heart Disease 9 and the Society of Thoracic Surgeons database. ${ }^{13}$ The latter study compared the early outcomes of isolated $\mathrm{CABG}$ surgeries from units that had a residency training program compared with units that did not have a residency program. They found no difference in adjusted early mortality, stroke, or renal failure but higher rates of "any reoperation," prolonged ventilation, and prolonged length of stay.

There are some limitations to this study. First, it is an observational study and thus may not have adequately adjusted for the case mix despite the use of propensity score methods. Second, we did not treat the allocation of surgeries on an intent-to-treat basis. Hence, cases in which the trainee started the surgery but it was completed by the surgeon due to problems arising early in the course of the surgery would almost certainly have been listed as a consultant surgery. However, this can be seen as a real-world assessment of the current system of training, which maintains safe patient outcomes. Third, the definition of a trainee surgery is in some surgeries imprecise. There would have been a minority of surgeries where the surgeon may have performed 1 out of 3 or more distal coronary anastamoses. This would have been variably listed as a trainee surgery depending on the individual trainee's perception of the surgery. Furthermore, where the trainee had performed the entire surgery, the degree of consultant supervision could have varied from the consultant being scrubbed and assisting the trainee throughout the surgery to surgeries where the consultant was available but not present in the operating theater. Finally, we have only studied survival as a midterm outcome. The study of other midterm outcomes such as freedom from revascularization or return of angina would be desirable.

In conclusion, $\mathrm{CABG}$ can be performed safely by trainee surgeons within a supervised training program with acceptable short- and midterm outcomes.

The Australasian Society of Cardiac and Thoracic Surgeons (ASCTS) Cardiac Surgery Database is an initiative of the ASCTS and is funded by the Department of Human Services, Victoria.

\section{References}

1. Hargreaves DH. A training culture in surgery. BMJ. 1996;313:1635-9.

2. Birkmeyer JD, Stukel TA, Siewers AE, Goodney PP, Wennberg DE, Lucas FL. Surgeon volume and operative mortality in the United States. $N$ Engl J Med. 2003;349:2117-27.

3. Adult Cardiac Surgery in New York State 2002-2004. Available at http:// www.health.state.ny.us/diseases/cardiovascular/heart_disease/docs/cabg_2002-2004. pdf. Accessed March 22, 2008.

4. Keogh B, Spiegelhalter D, Bailey A, Roxburgh J, Magee P, Hilton C. The legacy of Bristol: public disclosure of individual surgeons' results. BMJ. 2004;329. 450-4.

5. DeLaRosa J, Thourani VH, Wheatley GH 3rd, McMullan DM, Karamanoukian RL, Greene MG, et al. Impact of resident duty hour standards 
on cardiothoracic residents and program directors. Ann Thorac Surg. 2005;80: 1569-71.

6. Oo AY, Grayson AD, Rashid A. Effect of training on outcomes following coronary artery bypass graft surgery. Eur J Cardiothorac Surg. 2004;25:591-6.

7. Jenkins DP, Valencia O, Smith EE. Risk stratification for training in cardiac surgery. Thorac Cardiovasc Surg. 2001;49:75-7.

8. Baskett RJ, Buth KJ, Legare JF, Hassan A, Hancock Friesen C, Hirsch GM, et al. Is it safe to train residents to perform cardiac surgery? Ann Thorac Surg. 2002;74: 1043-8.

9. Sethi GK, Hammermeister KE, Oprian C, Henderson W. Impact of resident training on postoperative morbidity in patients undergoing single valve replacement. Department of Veterans Affairs Cooperative Study on Valvular Heart Disease. J Thorac Cardiovasc Surg. 1991;101:1053-9.

10. Caputo M, Chamberlain MH, Ozalp F, Underwood MJ, Ciulli F, Angelini GD. Off-pump coronary operations can be safely taught to cardiothoracic trainees. Ann Thorac Surg. 2001;71:1215-9.

11. Goodwin AT, Birdi I, Ramesh TP, Taylor GJ, Nashef SA, Dunning JJ, et al. Effect of surgical training on outcome and hospital costs in coronary surgery. Heart. 2001;85:454-7.

12. Gulbins H, Pritisanac A, Ennker IC, Ennker J. Safety of a cardiac surgical training program over a twelve-year period. Thorac Cardiovasc Surg. 2007; 55:494-9.
13. Haan CK, Milford-Beland S, O'Brien S, Mark D, Dullum M, Ferguson TB, et al. Impact of residency status on perfusion times and outcomes for coronary artery bypass graft surgery. Ann Thorac Surg. 2007;83:2103-10.

14. Reid CM, Rockell M, Skillington PD, Shardey GC, Smith JA, Yii M, et al. Initial twelve months experience and analysis for 2001-2002 from the Australasian Society of Cardiac and Thoracic Surgeons-Victorian database project. Heart Lung Circ. 2004;13:291-7.

15. ASCTS Cardiac Surgery Database Project Data Definitions Version 1 30/04/01. Available at http://www.ascts.org/documents/PDF/definitions.pdf. Accessed on March 22, 2008.

16. Dinh DT, Lee GA, Billah B, Smith JA, Shardey GC, Reid CM. Trends in coronary artery bypass graft surgery in Victoria, 2001-2006: findings from the Australasian Society of Cardiac and Thoracic Surgeons database project. Med J Aust. 2008; 188:214-7.

17. Alderman EL, Levy JH, Rich JB, Nili M, Vidne B, Schaff H, et al. Analyses of coronary graft patency after aprotinin use: results from the International Multicenter Aprotinin Graft Patency Experience (IMAGE) trial. J Thorac Cardiovasc Surg. 1998;116:716-30.

18. Casati V, Guzzon D, Oppizzi M, Bellotti F, Franco A, Gerli C, et al. Tranexamic acid compared with high-dose aprotinin in primary elective heart operations: effects on perioperative bleeding and allogeneic transfusions. J Thorac Cardiovasc Surg. 2000;120:520-7. 